\title{
Intranasal Oxytocin Increases Perceptual Salience of Faces in the Absence of Awareness
}

\author{
Shao-Wei Xue ${ }^{1,2 *}$, Hua-Bo $\mathrm{Wu}^{1 *}$, Lanhua Zhang ${ }^{3}$, and De-Xuan Zhang ${ }^{1 凶}$ \\ ${ }^{1}$ Center for Cognition and Brain Disorders, Institutes of Psychological Sciences and the Affiliated Hospital, Hangzhou Normal University, \\ Hangzhou, China \\ ${ }^{2}$ Zhejiang Key Laboratory for Research in Assessment of Cognitive Impairments, Hangzhou, China \\ ${ }^{3}$ College of Medical Information and Engineering, Shandong First Medical University \& Shandong Academy of Medical Sciences, Tai'an, China
}

Objective The neuropeptide oxytocin has been found to improve human social cognition and promote prosocial behavior. However, it is still unclear about the mechanisms underlying these effects of oxytocin on neural processes, such as visual perception and awareness. Especially, it is still unclear whether oxytocin influences perceptual salience of social stimuli in the absence of awareness.

Methods In a randomized double-blind, placebo-controlled trial we applied an interocular suppression paradigm and eye tracking methods to investigate the influence of intranasally administered oxytocin on perceptual salience of social stimuli. Suppression times and pupillometric data were measured during subjects being presented with gradually introduced pictures of social stimuli (neutral expression faces) or nonsocial stimuli (grayscale watch pictures) that were suppressed and invisible in 10 men who were administered 24 IU oxytocin and 10 men who were administered a placebo.

Results The results demonstrated that the oxytocin group perceived social stimuli more quickly accompanied by subsequent larger increasing pupil diameter than nonsocial stimuli, indicating an increased unconscious salience of social stimuli.

Conclusion These findings provided new insights into oxytocin's modulatory role to social information processing, suggesting that oxytocin might enhance attentional bias to social stimuli even after removal of awareness.

Psychiatry Investig 2020;17(4):292-298

Key Words Oxytocin, Face, Unconscious, Pupil diameter.

\section{INTRODUCTION}

The neuropeptide oxytocin has recently received considerable attention as a potential approach to promote social cognition and prosocial behavior in humans. ${ }^{1-3}$ For example, previous studies have reported that intranasally administered oxytocin can increase emotion recognition ${ }^{4-6}$ and empathy, ${ }^{7-9}$ improve the salience of social cues ${ }^{10}$ and especially facial expressions, ${ }^{11-13}$ promote trust perceptions and trust behavior, ${ }^{14}$ and reduce social stress and anxiety. ${ }^{15-18}$ Oxytocin is famous for facilitatory role in parturition and lactation as a hormone

Received: May 21, 2019 Revised: September 20, 2019

Accepted: December 7, 2019

$\triangle$ Correspondence: De-Xuan Zhang, PhD

Center for Cognition and Brain Disorders, Hangzhou Normal University, 2318

Yuhangtang Road, Hangzhou, Zhejiang 311121, China

Tel: +86-571-28867717, Fax: +86-571-28867717

E-mail: dexuan.zhang@gmail.com

*These authors contributed equally to this work.

(a) This is an Open Access article distributed under the terms of the Creative Commons Attribution Non-Commercial License (https://creativecommons.org/licenses/by$\mathrm{nc} / 4.0$ ) which permits unrestricted non-commercial use, distribution, and reproduction in any medium, provided the original work is properly cited. synthesized by the hypothalamus, ${ }^{3,19}$ and has been also reported to affect the activation of some brain regions, such as the nucleus accumbens in the ventral striatum, ${ }^{20}$ amygdala, ${ }^{21-23}$ and midbrain regions. ${ }^{14}$ The region-specific distribution of oxytocin effects might be associated with the regulation of social cognition and behavior. ${ }^{24}$ However, it is still unclear about the mechanism underlying these effects of oxytocin on neural processes such as visual perception and awareness.

Attention and consciousness are known as separable psychological processes that usually occur together. ${ }^{25}$ Previous studies have shown that attention modulated visual perception and even the effects of stimuli that were suppressed and invisible. ${ }^{26}$ Understanding the mechanisms in processing invisible visual stimuli will bring new insights into how the visual system operates under conditions of unconsciousness and even the neural correlates of consciousness. ${ }^{27}$ Oxytocin could increase the individual's attention to social agents. ${ }^{21,28}$ These effects on attentional modulation might be pivotal for oxytocin as an intervention tool in promoting social-cognitive functioning, especially for individuals with joint-attention deficits 
in autism ${ }^{1,8,12,19,29,30}$ and social phobia. ${ }^{29}$ However, the underlying mechanism has not yet been investigated in the absence of perceptual awareness and it is still not known whether the modulation has to involve in conscious awareness.

The pupil size of the human eye is often used as a sensitive and reliable indicator of the physiological arousal state, because it is assumed that the pupil size is related to the amount of attention and other cognitive processes. For example, larger pupil diameter indicates greater processing load. ${ }^{31}$ Pupillometry in psychology measures pupil diameter. The changes in pupil diameter are controlled by two muscles (the dilator and sphincter) that are innervated by activity in the sympathetic and parasympathetic branches of the nervous system. ${ }^{5}$ Previous studies have provided evidences that intranasal oxytocin influenced social stimulus-induced pupil dilation, reflecting effects on attention modulation. ${ }^{5,32}$ However, oxytocin's effects on pupillary responses to social stimuli without conscious awareness have not yet been investigated.

In the present study, our main objective was to establish whether intranasal oxytocin related to enhanced perception salience of social stimuli under conditions of unawareness compared to nonsocial stimuli. Binocular rivalry offers a particularly elegant way by presenting continuous flashing of random Mondrian images (around $10 \mathrm{~Hz}$ ) to one eye to suppress a visual stimulus to the other eye from awareness. ${ }^{27,33-35} \mathrm{We}$ therefore used a double-blind, placebo-controlled design, with subjects being presented with pictures of social or nonsocial stimuli during binocular suppression after intranasal administration of 24 IU oxytocin or placebo. Observers viewed a composite of dynamic red Mondrain patterns and the green social or nonsocial stimuli through red-green anaglyph glasses, rendering the test images invisible. Suppression times and pupillometric data were measured to describe how subjects gained dominance against the suppression noise to perceive gradually introduced test stimuli. We hypothesized that the oxytocin group had spent less time to gain dominance against the suppression noise accompanied by subsequent larger increasing pupil diameter for the social stimuli compared with the nonsocial stimuli.

\section{METHODS}

\section{Participants}

Twenty healthy Chinese male undergraduates at Hangzhou Normal University (mean age, 21.7 years; SD, 1.7 years) were randomly assigned to an experimental group (oxytocin; $n=10$ ) and a control group (placebo; $\mathrm{n}=10$ ). All participants had normal or corrected-to-normal vision, did not take any medication that could influence the pupillary response, reported no history of endocrine, neurological or mental disorder, and were instructed to abstain from alcohol or caffeinated drinks, and nicotine on the day of the study. The study procedures were conducted in accordance with the Declaration of Helsinki and were approved by the local Institutional Review Boards (IRB number: 20130609) in Hangzhou Normal University. All subjects provided written, IRB-approved consent before participating.

\section{Oxytocin administration}

Participants self-administered a single intranasal dose of 24 IU oxytocin (Syntocinon Spray, Meike; three puffs per nostril, each puff containing $4 \mathrm{IU}$ oxytocin) or placebo (containing only carrier substance). Previous studies have indicated that single-dose intranasal administration (a 24 IU dose) of oxytocin enabled direct access of the peptide to the central nervous compartment ${ }^{36}$ with no adverse side-effects being reported ${ }^{37}$ thus providing a useful approach for investigating oxytocin circuits within the human brain. ${ }^{10,38}$ Self-report after the experiment showed that participants were not able to discriminate whether they had received oxytocin or placebo. Participants also did not report any side effect following drug administration.

\section{Stimuli and procedure}

The experiment was conducted in a quiet and slightly dimmed room. Participants were seated comfortably in front of a 17" Cathode Ray Tube (CRT) monitor with a resolution of $1024 \times 768$ pixels and a refresh rate of $100 \mathrm{~Hz}$ at a distance of $57 \mathrm{~cm}$. Visual stimuli were presented with Matlab (The MathWorks, Natick, MA, USA), using the Cogent 2000 toolbox. During presentation, subjects had their heads fixated on a headrest.

A total of 90 grayscale Chinese face images with neutral expressions (45 men and 45 women) were chosen as simple social stimuli from the Chinese Affective Picture System (CAPS). Nonsocial stimuli were 90 grayscale watch pictures. The experiment contained 270 trials, divided into three blocks with 90 trials each ( 45 faces and 45 nonsocial stimuli). They were presented in a mask on a black background (Figure 1B). Two black frames $\left(8.6^{\circ} \times 8.6^{\circ}\right)$ were presented side by side on the screen, such that one frame was only visible to one eye during the whole experiment. A black central fixation cross $\left(0.8^{\circ} \times 0.8^{\circ}\right)$ was always displayed in the center of each frame. Participants were asked to maintain stable fixation throughout each experimental block. Each trial began with a $500-\mathrm{ms}$ presentation of the fixation cross. The high-contrast colored Mondrian-like continuous flash suppression (CFS) masks were flashed to one eye at a frequency of $10 \mathrm{~Hz}$, and the test image $\left(2.4^{\circ} \times 2.7^{\circ}\right)$ was presented to the other eye in a random position either to the left or to the right of the central fixation cross, at a random lo- 
cation within the area corresponding to the location of the CFS masks. The horizontal distance between the center of the test image and the fixation cross ranged from $2.1^{\circ}$ to $2.9^{\circ}$, and the vertical center of the test image was anywhere between $2.9^{\circ}$ above and $2.9^{\circ}$ below the fixation cross. The suppressed eyes were balanced in a pseud-random order. To ensure that the suppression noise was the dominant percept at the beginning of each trial, the dynamic CFS masks were presented im-

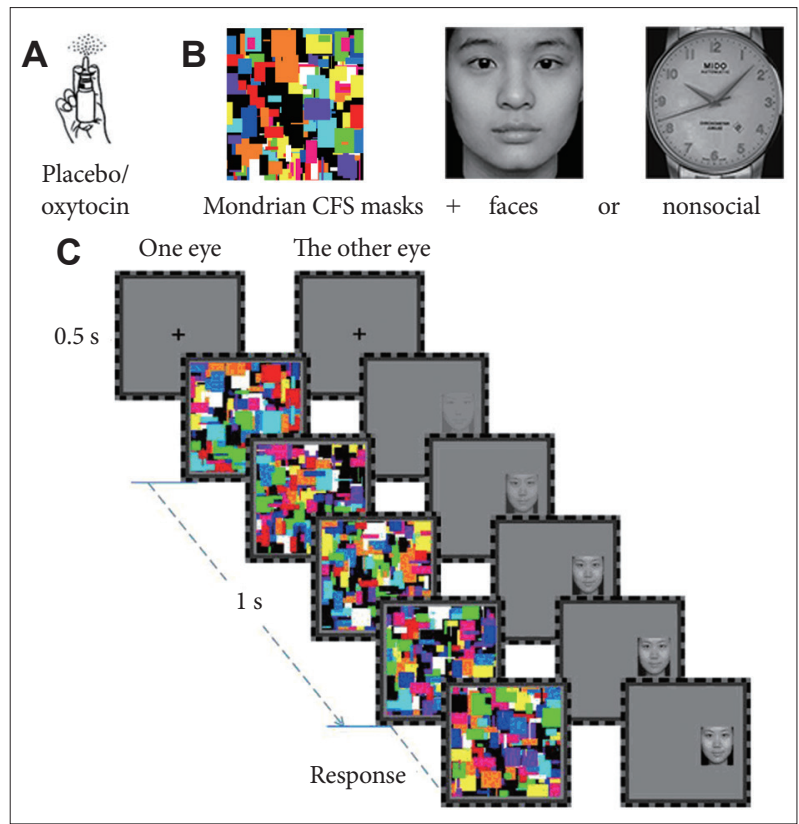

Figure 1. Experimental procedure with examples of social (faces) and nonsocial stimuli. A: Intranasal oxytocin and placebo administration. B: Three types of stimuli images (mask stimuli: Mondrianlike CFS, test stimuli: Faces and nonsocial stimuli). C: Schematic illustration of a single trial. In the invisible condition, the test images presented to one eye can be completely suppressed from awareness by dynamic Mondrian patterns presented to the other eye. CFS: continuous flash suppression. mediately at full contrast. The test image appeared 0, 100, 200, 300 , or $400 \mathrm{~ms}$ after the CFS masks displayed. The contrast of the test stimulus was ramped up gradually from 0 to $100 \%$ (to its original contrast) within a period of $1 \mathrm{~s}$ and then remained constant until the participant had made a keyboard response, or disappeared after $4 \mathrm{~s}$ if the participant got keyboard response. At the very beginning of each trial, participants only perceived the CFS noise patch and were unaware of which side contained the test image. To measure the response time taken for the test image to overcome the suppression noise and become dominant, participants were asked to judge whether any part of the test image appeared at the right or left of the fixation point and respond with left or right key. They did not need to know the specific content of the image. At the end of each trial was a fixation cross presented for 1,500 ms centered in the black frame. Figure 1 shows the general paradigm for the two experimental conditions.

\section{Pupil data acquisition}

Pupil diameter was continuously recorded using an Applied Science Laboratory (ASL) D6 desk-mounted optics remote eye-tracking system. The pan/tilt module, a component of the ASL system, used infrared technology to illuminate the eye and telephoto an image of the eye onto an eye camera. The D6 control unit then extracted the pupil and reflection of the light source on the cornea and computed pupil diameter at a sampling rate of $60 \mathrm{~Hz}$. The changes in pupil diameter might be due to changes in lighting conditions (i.e., differences in either illumination of the room or luminance of the experimental stimuli), and therefore we kept illumination in the laboratory constant for all stimuli and the cumulative brightness of the pictures between the conditions ensuring that luminance of the picture stimuli did not differ systematically. ${ }^{5}$

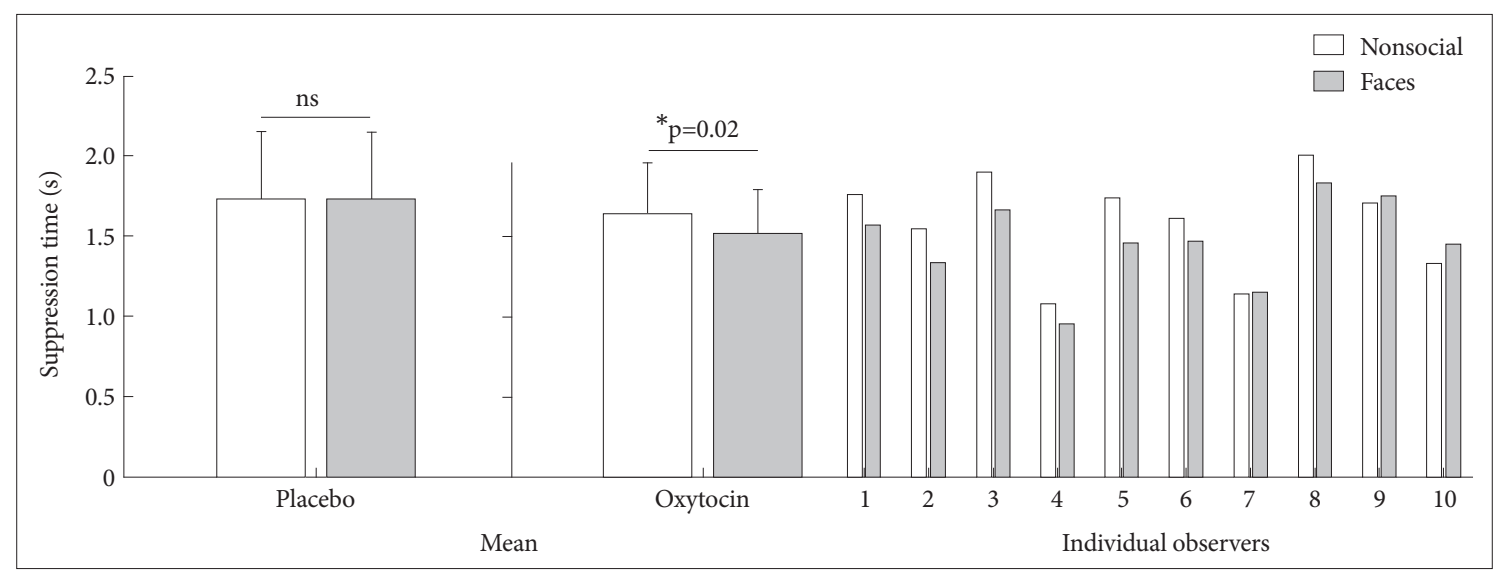

Figure 2. Suppression times for faces and nonsocial stimuli in the placebo and oxytocin group. The two bars on the left show the suppression times in the placebo condition, in which the test images are presented binocularly on the noise background. The right side of the figure shows the suppression times for 10 individual observers in the oxytocin group, as well as the averaged suppression times. Error bars represent standard error of the mean. Asterisks indicate a significant difference between faces and nonsocial stimuli. ${ }^{*} p<0.05$. 


\section{Statistical analysis}

We tested whether oxytocin application had an effect on suppression times for the face and nonsocial stimuli and pupillary responses (average pupil diameter changes). Statistical analyses relied on ANOVA, which included a group factor drug (placebo versus oxytocin); a repeated measures factor stimulus category (nonsocial stimuli versus faces). Statistical analyses were performed using SPSS 20 (IBM Corp., Armonk, NY, USA).

\section{RESULTS}

\section{Behavioral results}

Suppression times were assigned into a 2 (Drug: Placebo, Oxytocin) $\times 2$ (Stimulus: Nonsocial stimuli, Face) ANOVA (Figure 2). The main effect of stimulus was significant [F (1, 18) $=6.019 ; \mathrm{p}=0.025]$, and the interaction effect of drug and stimulus was also significant $[\mathrm{F}(1,18)=6.44 ; \mathrm{p}=0.021]$. We found no main effect of drug $[F(1,18)=0.925 ; p=0.349]$. Subsequent $\mathrm{t}$ tests indicated that suppression times of faces was significantly faster than those of nonsocial stimuli in the oxyto-
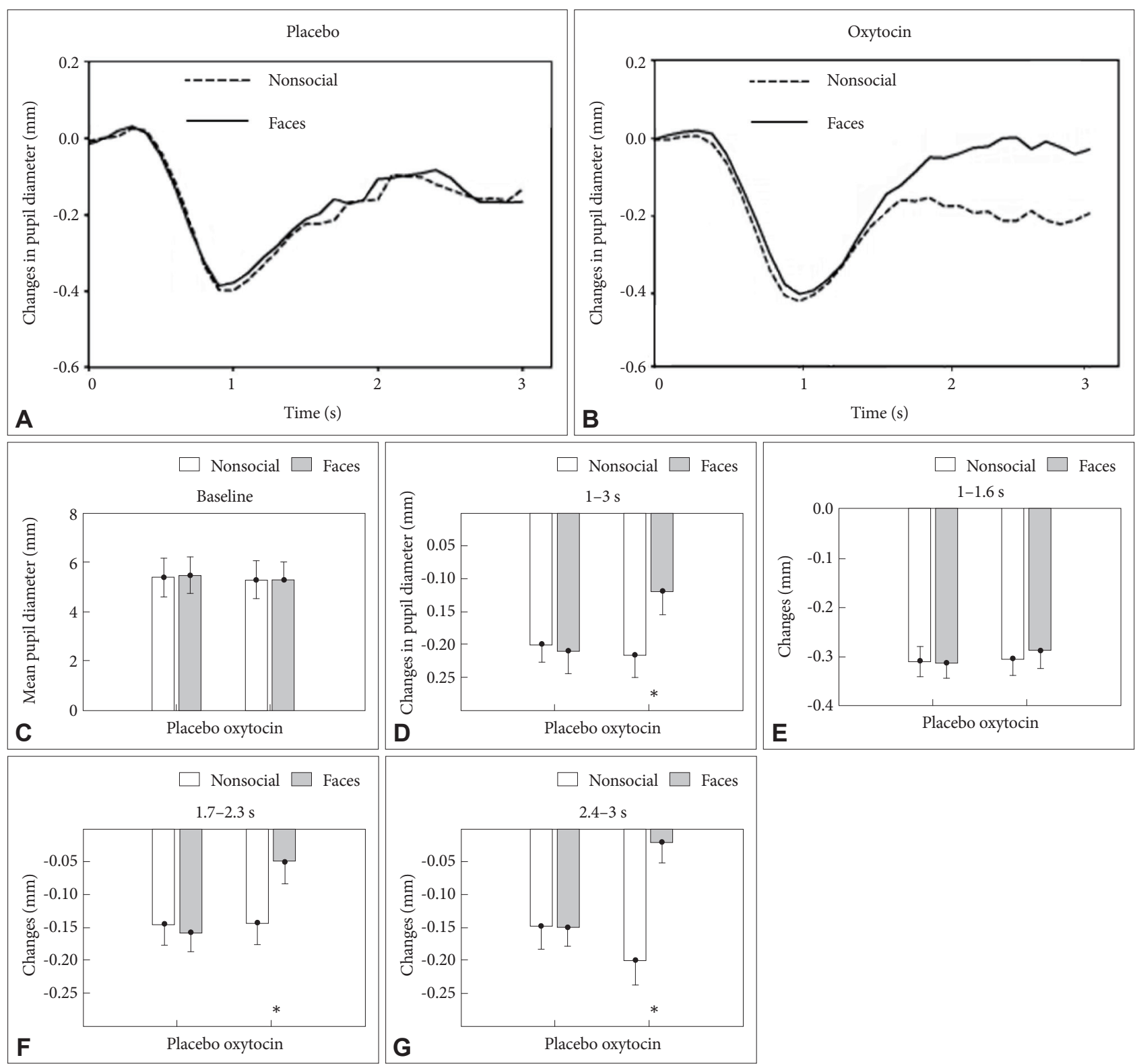

Figure 3. Changes of pupil diameter in the face and nonsocial stimuli conditions for the placebo (A) and oxytocin (B) group. (C) Baseline pupil diameter. Average changes of pupil diameter between 1 and $3 \mathrm{~s}(\mathrm{D})$, between 1 and $1.6 \mathrm{~s}(\mathrm{E})$, between 1.7 and $2.3 \mathrm{~s}(\mathrm{~F})$ and between 2.4 and $3 \mathrm{~s}(\mathrm{G})$. Error bars indicate standard error of the mean, ${ }^{*} \mathrm{p}<0.05$. 
cin group [ $\mathrm{t}(9)=-2.822 ; \mathrm{p}=0.02]$ but not in the placebo group [ $\mathrm{t}(9)=0.090 ; \mathrm{p}=0.93]$.

\section{Pupil results}

The baseline pupil diameter before the presentation of the first picture of each condition was analyzed by the same manner as behavioral results reported above (Figure 3C). The main effect of drug on baseline pupil diameter was not significant $[\mathrm{F}(1,18)=0.154 ; \mathrm{p}=0.701]$, and neither an effect of stimulus [ $\mathrm{F}$ $(1,18)=0.83 ; \mathrm{p}=0.5]$ nor of Drug $\times$ Stimulus interaction $[\mathrm{F}(1$, 18) $=0.02 ; p=0.92]$ was also significant. The results indicated that the following differences in the task-related pupil diameter did not result from differences of baseline pupil diameter.

As shown in Figure 3A and B, during the period of $1 \mathrm{~s}$ after the beginning of each trial, pupil diameter decreased monotonously in both conditions in the oxytocin and placebo group, indicating a shrink of eye pupil when trying gaining dominance against the suppression noise to perceive the test stimuli. In keeping with the above behavioral results, the curve of pupil diameter was reversed about after $1 \mathrm{~s}$, and then increased with the presentation of the subsequent pictures of the test stimuli over the course of the trial in the both conditions and groups, reflecting increased resource allocation during perceptual processing. Moreover, subsequent pupil dilations were always greater for the face stimuli than for the nonsocial stimuli in the oxytocin group, but not in the placebo group; the observed changes in pupil diameter suggested that greater allocation of cognition resources for social stimuli was associated with oxytocin application.

ANOVAs were conducted with groups (oxytocin and placebo) and stimulus conditions (face and nonsocial stimuli) as factors, using each of average changes of pupil diameter in the interval of interest between 1 and $3 \mathrm{~s}$ as dependent variables. The main effect of stimulus was significant $[\mathrm{F}(1,18)=8.477$; $\mathrm{p}=0.01$ ], and the interaction effect of drug and stimulus was also significant $[\mathrm{F}(1,18)=4.84 ; \mathrm{p}=0.042]$. We found no main effect of drug $[F(1,18)=2.453 ; p=0.136]$ (Figure 3D). The interval of interest was further divided into three sub-periods: $1-1.6 \mathrm{~s}, 1.7-2.3 \mathrm{~s}$, and $2.4-3 \mathrm{~s}$. The face stimuli had significant larger mean change of pupil diameter than that of the nonsocial stimuli $(\mathrm{p}<0.05)$ for $1.7-2.3 \mathrm{~s}$ (Figure $3 \mathrm{~F}$ ) and for 2.4-3 s (Figure 3G), but not for 1-1.6 s (Figure 3E).

\section{DISCUSSION}

In the present study, we applied an interocular suppression paradigm and eye tracking methods to investigate whether intranasal oxytocin could modulate perceptual salience of social stimuli that were suppressed and invisible. The results showed that the oxytocin group spent less time to gain dom- inance against the suppression noise accompanied by subsequent larger increasing pupil diameter for the social stimuli compared with the nonsocial stimuli, whilst the placebo group had no significant difference, indicating that oxytocin could enhance perceptual salience of social stimuli under interocular suppression.

The placebo group showed no significant difference of suppression times between the two stimuli; this ruled out the probability that the two stimuli per se had different ability to break suppression. A previous study 33 reported an upright face took less time than an upside-down face to gain dominance against the identical suppression noise during rivalry. In the present study, in contrast, the result of the oxytocin group implied that the suppressed and invisible faces (social stimuli) were processed more quickly against a noise background than the suppressed nonsocial stimuli. We therefore suggested that effects of oxytocin on information processing of social stimulus could cause the above unconscious differences. We argued one mechanism underlying the effects might be oxytocin-mediated attentional bias to social stimuli. In the invisible (suppressed) condition observers perceived the dynamic noise and were not aware of the test pictures presented. Although the information from the suppressed stimuli did not survive interocular competition at the cortical level, it might be processed subcortically with multistage competition and reached cortical areas along a subcortical pathway, ${ }^{39,40}$ for example, the retinocollicular-pulvinar-amygdala pathway, which proceeded from the retina to the superior colliculus, posterior nuclei of the thalamus, such as the pulvinar, and then onto the amygdala and other high-level brain areas. ${ }^{27}$ Oxytocin-mediated attention was regarded as a key factor of quickening social information processing in this pathway. Why attention failed to modulate neural activity for the nonsocial stimuli but succeeded in modulating neural activity for invisible faces? We provided an interpretation that oxytocin specially increased salience of the invisible social stimuli and strengthened the signal of their images, in turn the social information was served as a cue to guide attention and their processing was modulated by attention, then resulting in shortened suppression durations. ${ }^{33}$

Pupil dilation is a physiological marker and its modulation is related to several cognitive functions, such as the deployment of attention. ${ }^{41}$ Pupil diameters increased in both experimental conditions were observed after an initial shrink caused by the appearance of the first picture of each trial. The results were consistent with a study about effects of oxytocin on recognition of socioaffective stimuli, ${ }^{5}$ and also dynamically reflected the above findings about less suppression times owing to oxytocin application. After $1 \mathrm{~s}$ the oxytocin group showed always larger subsequent pupil dilation for the social stimuli than for the nonsocial stimuli, but the placebo group did not 
have these significantly different trends; this indicated that greater increased pupil dilation after intranasally administered oxytocin was associated with more allocation of attentional resource. ${ }^{31,42}$ Besides, pupil diameter in the baseline phase did not differ between groups and stimuli conditions; this ruled out the possibility that changes in pupil diameter were due to pre-experimental pupil baseline diameter in individuals. In the present study, in contrast, we observed differences in pupil diameter between social and nonsocial stimuli only in the oxytocin group.

Our findings are consistent with previous studies about the effects of oxytocin on human behavior and social cognition. Previous studies have reported that oxytocin was involved in allocating attentional resources to social stimuli. For example, oxytocin increased gaze to the eye region, which was regarded as the most socially communicative aspect of the face, ${ }^{1,28}$ and enhanced functional coupling of posterior amygdala and superior colliculi. Oxytocin has shown to reduce attention to negative social emotions, supporting that oxytocin served to inhibit withdrawal-related social behavior, ${ }^{43}$ while another study suggested that oxytocin increased covert attention to happy faces, and supported the hypothesis that oxytocin modulated early attentional processed that might promote prosocial behavior. ${ }^{44}$ An electroencephalographic (EEG) study has showed that oxytocin enhanced differential response of EEG rhythms in the mu/alpha and beta ranges during tasks about perception of biological versus nonbiological motion, suggesting the involvement of attentional mechanisms in brain activity. ${ }^{45}$ Some studies have tried to use short presentation times to assess oxytocin effects on recognition of emotional facial stimuli under conditions of limited awareness. ${ }^{4}$ Our study provided new insights into oxytocin's modulatory role to social information processing, suggesting that oxytocin might increase attentional bias to social stimuli even after removal of awareness. As persistent fear or avoidance of social interactions can be observed in some neuropsychiatric disorders such as autism spectrum disorder, social anxiety disorder, depression, and schizophrenia, our findings of the "pro-social" effects of oxytocin suggested that oxytocin might become a promising target for novel possible treatment approaches for these disorders characterized by social deficits.

There are some additional limitations in materials and methodology in the present study. We only tested a sample of male subjects, whereas studies with female subjects were complicated by the fact that estradiol, levels of which varied across the menstrual cycle, was known to induce oxytocin receptors and presumably increase responsively to oxytocin ${ }^{46}$ Since oxytocin appeared to have sex-dimorphic effects, ${ }^{47}$ our results might not be generalizable to women. For faces constituted the primary social cue and played a major role in carrying social in- formation, we used face stimuli as a social content, but we designed only simple stimuli with neutral expressions. Otherwise, the face information with high social or emotional significance might be involved in differences of social information. ${ }^{48}$ To further elucidate this socioaffective question, we recommend the use of face stimuli with emotional expressions and nonface social stimuli in further unconscious studies to untie affective and social aspects of the stimulus material. Another limitation of our study is the small sample size. A large sample is needed in future research to support the findings of the present study. Future research should continue to investigate the relation among oxytocin, visual attention, and social information by functional magnetic resonance imaging (fMRI) and EEG.

In summary, the present study demonstrated that a single dose of intranasal oxytocin enhanced perceptual salience of faces in the unconscious condition by binocular suppression. Increased pupil diameter in combination with improved face perception provided evidence for an oxytocin-induced recruitment of supplemental attentional resources during the processing of social stimuli. The results have showed oxytocin's modulatory role on attentional bias to social information. Future research should address the possible as an intervention tool for improvement of joint-attention deficits in autism.

\section{Acknowledgments}

This study was supported by the Natural Science Foundation of Zhejiang Province (LY17H180007), the Hangzhou Science and Technology Commission Foundation (20170533B06), the Scientific Research Start-up Foundation (PF15002004049) and the cultivation project of the province-leveled preponderant characteristic discipline in the College of Education (18JYXK036) of Hangzhou normal University.

\section{Conflicts of Interest}

The authors have no potential conflicts of interest to disclose.

\section{Author Contributions}

Conceptualization: De-Xuan Zhang. Data curation: Hua-Bo Wu. Formal analysis: Hua-Bo Wu. Funding acquisition: Shao-Wei Xue. Investigation: Shao-Wei Xue, Hua-Bo Wu. Methodology: Hua-Bo Wu. Project administration: De-Xuan Zhang. Resources: De-Xuan Zhang. Supervision: DeXuan Zhang. Validation: De-Xuan Zhang. Visualization: Hua-Bo Wu. Writing-original draft: Shao-Wei Xue, Lanhua Zhang. Writing-review \& editing: Shao-Wei Xue.

\section{ORCID iDs}

Shao-Wei Xue

Hua-Bo Wu

Lanhua Zhang

De-Xuan Zhang https://orcid.org/0000-0001-5441-4522

https://orcid.org/0000-0002-9482-5219

https://orcid.org/0000-0003-3895-1170

https://orcid.org/0000-0003-4460-7384

\section{REFERENCES}

1. Bartz JA, Zaki J, Bolger N, Ochsner KN. Social effects of oxytocin in humans: context and person matter. Trends Cogn Sci 2011;15:301-309.

2. Ishak WW, Kahloon M, Fakhry H. Oxytocin role in enhancing well-being: a literature review. J Affect Disord 2011;130:1-9. 
3. Donaldson ZR, Young LJ. Oxytocin, vasopressin, and the neurogenetics of sociality. Science 2008;322:900-904.

4. Schulze L, Lischke A, Greif J, Herpertz SC, Heinrichs M, Domes G. Oxytocin increases recognition of masked emotional faces. Psychoneuroendocrinology 2011;36:1378-1382.

5. Prehn K, Kazzer P, Lischke A, Heinrichs M, Herpertz SC, Domes G. Effects of intranasal oxytocin on pupil dilation indicate increased salience of socioaffective stimuli. Psychophysiology 2013;50:528-537.

6. Domes G, Heinrichs M, Michel A, Berger C, Herpertz SC. Oxytocin improves "mind-reading" in humans. Biol Psychiatry 2007;61:731-733.

7. Hurlemann R, Patin A, Onur OA, Cohen MX, Baumgartner T, Metzler $\mathrm{S}$, et al. Oxytocin enhances amygdala-dependent, socially reinforced learning and emotional empathy in humans. J Neurosci 2010;30:49995007.

8. Guastella AJ, Einfeld SL, Gray KM, Rinehart NJ, Tonge BJ, Lambert TJ, et al. Intranasal oxytocin improves emotion recognition for youth with autism spectrum disorders. Biol Psychiatry 2010;67:692-694.

9. Bartz JA, Zaki J, Bolger N, Hollander E, Ludwig NN, Kolevzon A, et al. Oxytocin selectively improves empathic accuracy. Psychol Sci 2010;21: 1426-1428.

10. Rimmele U, Hediger K, Heinrichs M, Klaver P. Oxytocin makes a face in memory familiar. J Neurosci 2009;29:38-42.

11. De Dreu CK, Greer LL, Van Kleef GA, Shalvi S, Handgraaf MJ. Oxytocin promotes human ethnocentrism. Proc Natl Acad Sci U S A 2011; 108:1262-1266.

12. Domes G, Heinrichs M, Kumbier E, Grossmann A, Hauenstein K, Herpertz SC. Effects of intranasal oxytocin on the neural basis of face processing in autism spectrum disorder. Biol Psychiatry 2013;74:164-171.

13. Van IMH, Bakermans-Kranenburg MJ. A sniff of trust: meta-analysis of the effects of intranasal oxytocin administration on face recognition, trust to in-group, and trust to out-group. Psychoneuroendocrinology 2012;37:438-443.

14. Baumgartner T, Heinrichs M, Vonlanthen A, Fischbacher U, Fehr E. Oxytocin shapes the neural circuitry of trust and trust adaptation in humans. Neuron 2008;58:639-650.

15. Labuschagne I, Phan KL, Wood A, Angstadt M, Chua P, Heinrichs M, et al. Oxytocin attenuates amygdala reactivity to fear in generalized social anxiety disorder. Neuropsychopharmacology 2010;35:2403-2413.

16. Linnen AM, Ellenbogen MA, Cardoso C, Joober R. Intranasal oxytocin and salivary cortisol concentrations during social rejection in university students. Stress 2012;15:393-402.

17. Eckstein M, Scheele D, Weber K, Stoffel-Wagner B, Maier W, Hurlemann R. Oxytocin facilitates the sensation of social stress. Hum Brain Mapp 2014;35:4741-4750.

18. Grimm S, Pestke K, Feeser M, Aust S, Weigand A, Wang J, et al. Early life stress modulates oxytocin effects on limbic system during acute psychosocial stress. Soc Cogn Affect Neurosci 2014;9:1828-1835.

19. Andari E, Duhamel JR, Zalla T, Herbrecht E, Leboyer M, Sirigu A. Promoting social behavior with oxytocin in high-functioning autism spectrum disorders. Proc Natl Acad Sci U S A 2010;107:4389-4394.

20. Dolen G, Darvishzadeh A, Huang KW, Malenka RC. Social reward requires coordinated activity of nucleus accumbens oxytocin and serotonin. Nature 2013;501:179-184.

21. Gamer M, Zurowski B, Buchel C. Different amygdala subregions mediate valence-related and attentional effects of oxytocin in humans. Proc Natl Acad Sci U S A 2010;107:9400-9405.

22. Rupp HA, James TW, Ketterson ED, Sengelaub DR, Ditzen B, Heiman JR. Amygdala response to negative images in postpartum vs nulliparous women and intranasal oxytocin. Soc Cogn Affect Neurosci 2014;9:48-54.

23. Riem MM, Bakermans-Kranenburg MJ, Pieper S, Tops M, Boksem MA, Vermeiren RR, et al. Oxytocin modulates amygdala, insula, and inferior frontal gyrus responses to infant crying: a randomized controlled trial. Biol Psychiatry 2011;70:291-297.

24. Domes G, Heinrichs M, Glascher J, Buchel C, Braus DF, Herpertz SC. Oxytocin attenuates amygdala responses to emotional faces regardless of valence. Biol Psychiatry 2007;62:1187-1190.

25. Koch C, Tsuchiya N. Attention and consciousness: two distinct brain processes. Trends Cogn Sci 2007;11:16-22.

26. Sumner P, Tsai PC, Yu K, Nachev P. Attentional modulation of sensorimotor processes in the absence of perceptual awareness. Proc Natl Acad Sci U S A 2006;103:10520-10525.

27. Lin Z, He S. Seeing the invisible: the scope and limits of unconscious processing in binocular rivalry. Prog Neurobiol 2009;87:195-211.

28. Guastella AJ, Mitchell PB, Dadds MR. Oxytocin increases gaze to the eye region of human faces. Biol Psychiatry 2008;63:3-5.

29. Kirsch P, Esslinger C, Chen Q, Mier D, Lis S, Siddhanti S, et al. Oxytocin modulates neural circuitry for social cognition and fear in humans. J Neurosci 2005;25:11489-11493.

30. Parker KJ, Garner JP, Libove RA, Libove RA, Hyde SA, Hornbeak KB, et al. Plasma oxytocin concentrations and OXTR polymorphisms predict social impairments in children with and without autism spectrum disorder. Proc Natl Acad Sci U S A 2014;111:12258-12263.

31. Wierda SM, van Rijn H, Taatgen NA, Martens S. Pupil dilation deconvolution reveals the dynamics of attention at high temporal resolution. Proc Natl Acad Sci U S A 2012;109:8456-8460.

32. Leknes S, Wessberg J, Ellingsen DM, Chelnokova O, Olausson H, Laeng B. Oxytocin enhances pupil dilation and sensitivity to 'hidden' emotional expressions. Soc Cogn Affect Neurosci 2013;8:741-749.

33. Jiang Y, Costello P, He S. Processing of invisible stimuli: advantage of upright faces and recognizable words in overcoming interocular suppression. Psychol Sci 2007;18:349-355.

34. Watanabe M, Cheng K, Murayama Y, Ueno K, Asamizuya T, Tanaka K, et al. Attention but not awareness modulates the BOLD signal in the human V1 during binocular suppression. Science 2011;334:829-831.

35. Tong F, Meng M, Blake R. Neural bases of binocular rivalry. Trends Cogn Sci 2006;10:502-511.

36. Born J, Lange T, Kern W, McGregor GP, Bickel U, Fehm HL. Sniffing neuropeptides: a transnasal approach to the human brain. Nat Neurosci 2002;5:514-516.

37. Heinrichs M, Baumgartner T, Kirschbaum C, Ehlert U. Social support and oxytocin interact to suppress cortisol and subjective responses to psychosocial stress. Biol Psychiatry 2003;54:1389-1398.

38. MacDonald E, Dadds MR, Brennan JL, Williams K, Levy F, Cauchi AJ. A review of safety, side-effects and subjective reactions to intranasal oxytocin in human research. Psychoneuroendocrinology 2011;36:11141126.

39. Pasley BN, Mayes LC, Schultz RT. Subcortical discrimination of unperceived objects during binocular rivalry. Neuron 2004;42:163-172.

40. Fang F, He S. Cortical responses to invisible objects in the human dorsal and ventral pathways. Nat Neurosci 2005;8:1380-1385.

41. de Gee JW, Knapen T, Donner TH. Decision-related pupil dilation reflects upcoming choice and individual bias. Proc Natl Acad Sci U S A 2014;111:E618-E625.

42. Libby WL Jr, Lacey BC, Lacey JI. Pupillary and cardiac activity during visual attention. Psychophysiology 1973;10:270-294.

43. Kim YR, Oh SM, Corfield F, Jeong DW, Jang EY, Treasure J. Intranasal oxytocin lessens the attentional bias to adult negative faces: a double blind within-subject experiment. Psychiatry Investig 2014;11:160-166.

44. Domes G, Sibold M, Schulze L, Lischke A, Herpertz SC, Heinrichs M. Intranasal oxytocin increases covert attention to positive social cues. Psychol Med 2013;43:1747-1753.

45. Perry A, Bentin S, Shalev I, Israel S, Uzefovsky F, Bar-On D, et al. Intranasal oxytocin modulates EEG mu/alpha and beta rhythms during perception of biological motion. Psychoneuroendocrinology 2010;35:14461453.

46. Lambert B, Declerck CH, Boone C. Oxytocin does not make a face appear more trustworthy but improves the accuracy of trustworthiness judgments. Psychoneuroendocrinology 2014;40:60-68.

47. Lischke A, Gamer M, Berger C, Grossmann A, Hauenstein K, Heinrichs $\mathrm{M}$, et al. Oxytocin increases amygdala reactivity to threatening scenes in females. Psychoneuroendocrinology 2012;37:1431-1438.

48. Williams MA, Morris AP, McGlone F, Abbott DF, Mattingley JB. Amygdala responses to fearful and happy facial expressions under conditions of binocular suppression. J Neurosci 2004;24:2898-2904. 